\title{
Autoimmune Hepatitis and Immunoglobulin G4-Associated Autoimmune Hepatitis
}

\author{
Norihisa Yada ${ }^{a}$ Masatoshi Kudo ${ }^{a}$ Hobyung Chung ${ }^{b}$ Tomohiro Watanabe ${ }^{c}$ \\ ${ }^{a}$ Department of Gastroenterology and Hepatology, Kinki University School of Medicine, Osakasayama, \\ ${ }^{b}$ Department of Gastroenterology and Hepatology, Kobe City Medical Center General Hospital, Kobe, and \\ 'Department of Gastroenterology and Hepatology, Kyoto University Graduate School of Medicine, Kyoto, Japan
}

\section{Key Words}

Autoimmune hepatitis . Autoimmune pancreatitis .

Immunoglobulin G4

\begin{abstract}
Autoimmune hepatitis (AlH) is a disease that is characterized by the presence of autoantibodies and elevated levels of serum immunoglobulin $\mathrm{G}(\mathrm{lgG})$ and hepatic enzymes. Its characteristic findings in the liver include interface hepatitis, infiltration of lymphocytes and plasma cells, and rosette formation, and is treated with immunosuppressive drugs. Autoimmune pancreatitis, a pancreatic disease caused by an autoimmune mechanism, is associated with elevated levels of serum IgG4 and the infiltration of lgG4-positive cells into the pancreatic parenchyma, and it is occasionally accompanied by systemic features. This systemic inflammatory disease characterized by the infiltration of IgG4-positive plasma cells and elevated serum IgG4 levels was recently classified as an IgG4-related disease. A few studies have reported AlH cases with infiltrated IgG4-positive plasma cells in the liver, suggesting the involvement of $\operatorname{lgG} 4$ in the pathogenesis of $\mathrm{AlH}$. This feature was called IgG4-associated $\mathrm{AlH}$ and only two studies have been published. However, the diagnostic criteria of IgG4-associated AlH has not been defined and the epidemiology and clinical features remain uncertain. The de-
\end{abstract}

gree of IgG4-positive plasma cell infiltration in the liver was different in each article. The serum lgG4 level was not elevated in one study, whereas it was severely elevated in the other. Corticosteroid therapy normalized liver enzymes in both studies. Further studies are needed to define the epidemiological features or diagnostic criteria.

(c) 2013 S. Karger AG, Basel

\section{Introduction}

The serological features of autoimmune hepatitis (AIH) are the presence of antinuclear antibody (ANA), anti-smooth muscle antibody (SMA) and anti-type 1 liver-kidney microsomal antibody (anti-LKM-1), and elevated levels of serum immunoglobulin G (IgG) and hepatic enzymes. Characteristic findings in the liver include interface hepatitis, infiltration of lymphocytes and plasma cells, and rosette formation. AIH is treated with steroids and immunosuppressive drugs.

Autoimmune pancreatitis (AIP), a pancreatic disease caused by an autoimmune mechanism, is associated with elevated levels of serum IgG4 and the infiltration of IgG4positive cells into the pancreatic parenchyma, and it is occasionally accompanied by nonpancreatic features. AIP is known to be accompanied by multiple organ failure, and

\section{KARGER}

E-Mail karger@karger.com

www.karger.com/ddi
(C) 2013 S. Karger AG, Basel

0257-2753/13/0316-0415\$38.00/0
Masatoshi Kudo

Department of Gastroenterology and Hepatology

Kinki University School of Medicine

377-2 Ohno-higashi, Osakasayama, Osaka 589-8511 (Japan)

E-Mail m-kudo@med.kindai.ac.jp 


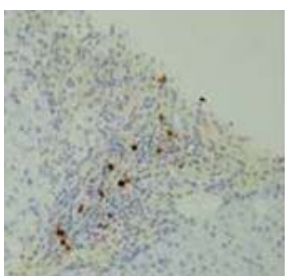

$\mathrm{AIH}$

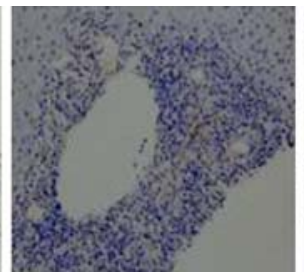

PBC

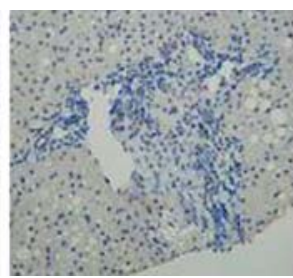

$\mathrm{HCV}$

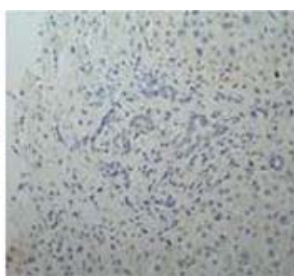

PSC

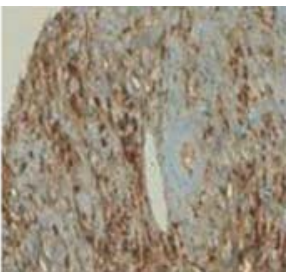

IgG4-associated cholangitis

Fig. 1. Immunohistochemical staining of IgG4. From left to right: IgG4-associated AIH, primary biliary cirrhosis, chronic hepatitis C, primary sclerosing cholangitis and IgG4-related sclerosing cholangitis. From Chung et al. [9].

Fig. 2. Comparison of PSL-induced changes in the serum ALT levels between IgG4associated AIH and IgG4-nonassociated AIH. Serum levels of ALT were monitored in 6 patients with IgG4-associated $\mathrm{AIH}$ and in 16 patients with IgG4-nonassociated AIH. These patients were treated with PSL. The doses of PSL at each time point are shown in the bottom panel. The results are shown as mean \pm standard error $\left({ }^{*} \mathrm{p}<0.05\right)$. From Chung et al. [9].

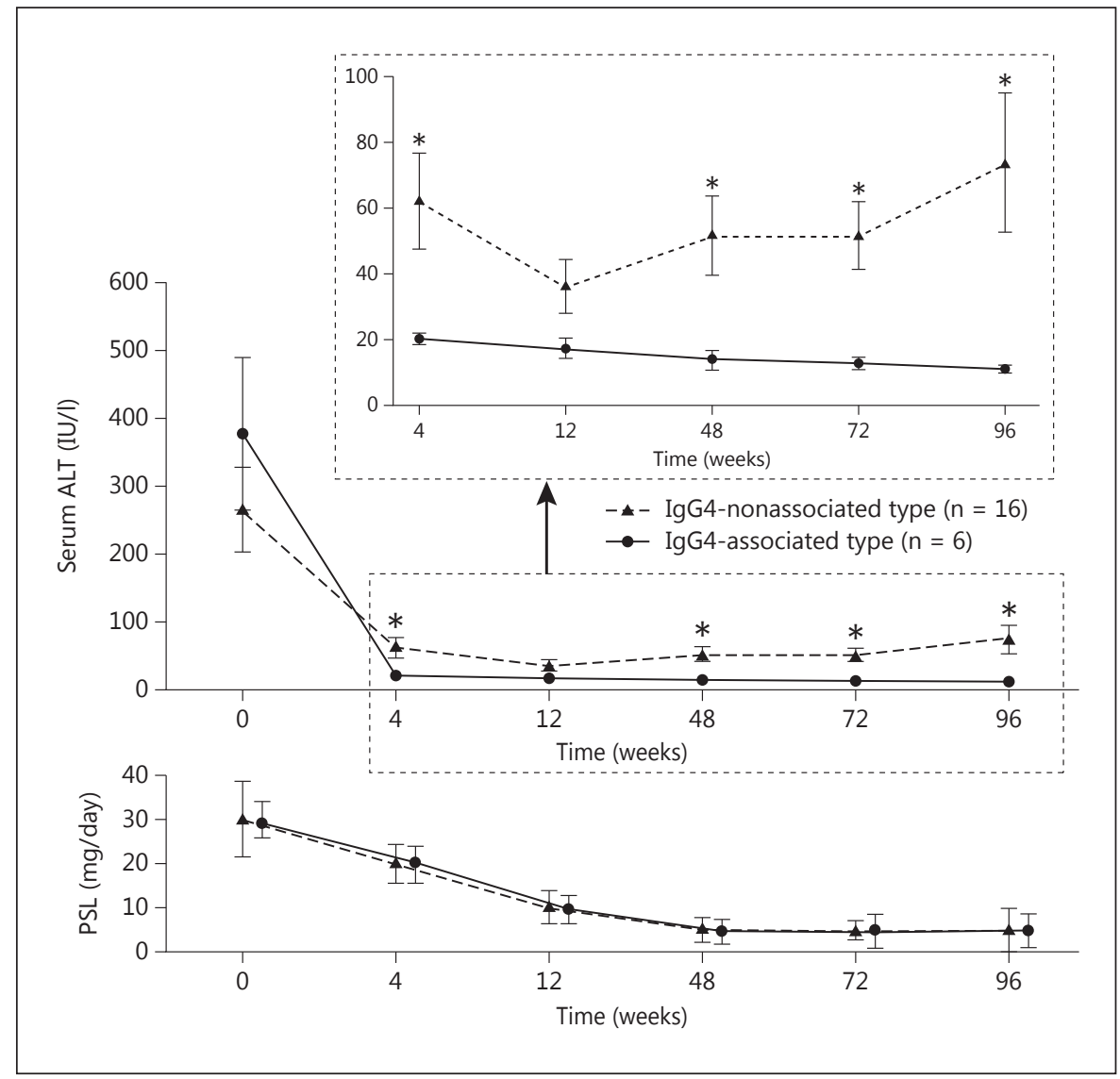

\section{Autoimmune Hepatitis}

systemic inflammatory diseases characterized by the infltration of IgG4-positive plasma cells and elevated serum IgG4 levels were recently classified as IgG4-related diseases.

Recent studies have reported AIH cases with infiltrated IgG4-positive plasma cells in the liver, suggesting the involvement of IgG4 in the pathogenesis of AIH. Here, we review the characteristic features of IgG4-related disease and its association with AIH.

\section{Clinical Features}

The term 'autoimmune hepatitis' was introduced in 1965 [1]. Upon the discovery of hepatitis C virus in 1989, chronic hepatitis $C$ infection was separated from non-A, non-B hepatitis. Subsequently, AIH was classified as an independent disease category, and AIH diagnostic crite- 
Table 1. Japanese diagnostic criteria for AIH in 1996

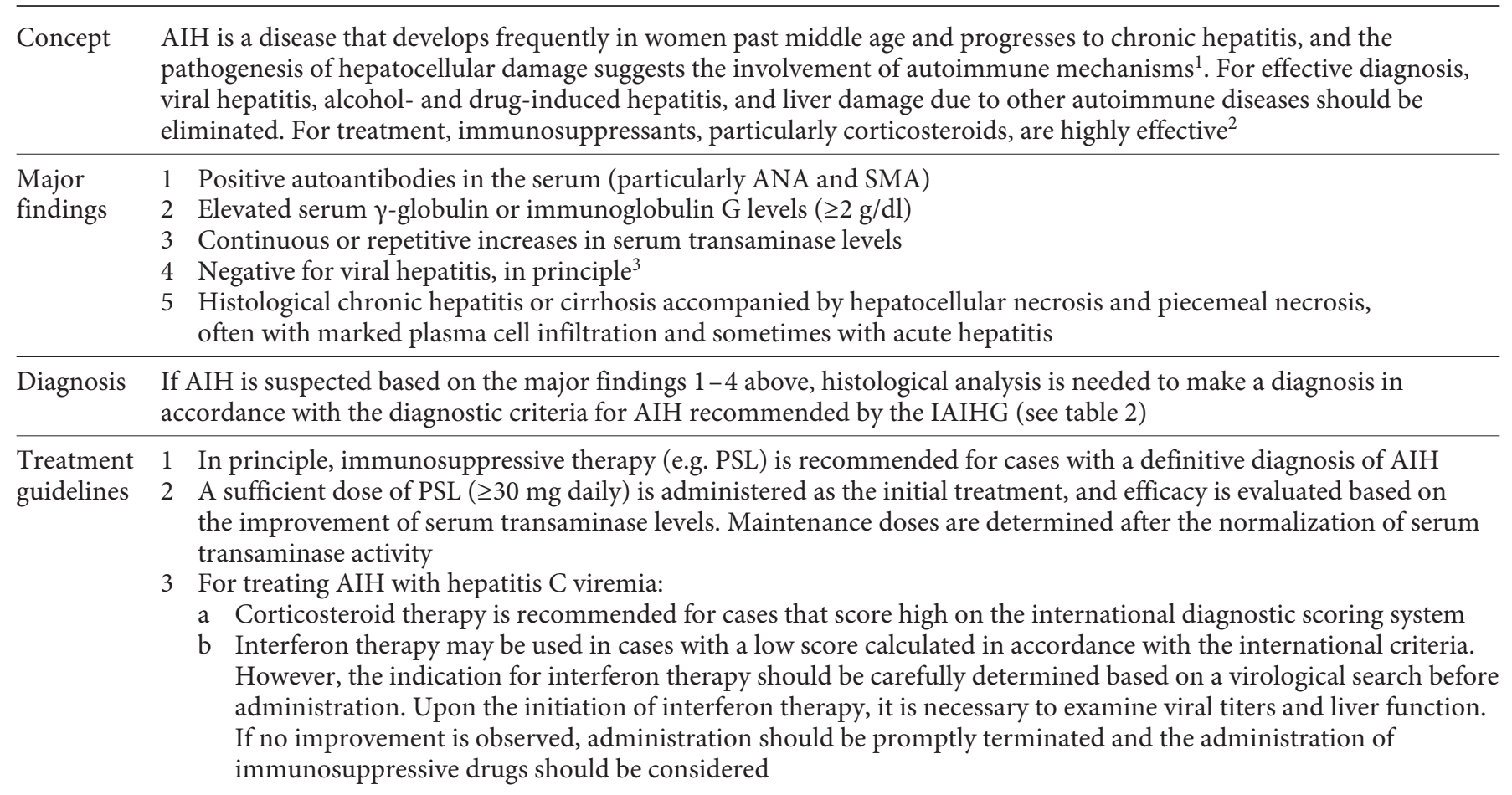

Modified from [4]. ${ }^{1}$ Human leukocyte antigen-DR4-positive cases are more common in Japan. ${ }^{2}$ Interferon therapy may be effective in some AIH cases with obvious hepatitis $\mathrm{C}$ infection. ${ }^{3}$ Some AIH cases are accompanied by hepatitis $\mathrm{C}$ viremia in Japan.

ria were established at the meeting of the International Autoimmune Hepatitis Group (IAIHG) in 1993 [2].

There is often a history of other autoimmune disorders in the patient or first-degree relatives. The disease predominates among women, the archetypal patient being a young female with endocrine abnormalities, but it also affects males and it can present at almost any age. Distribution of age at onset was thought to be bimodal, with peaks around puberty and between the fourth and sixth decades of life, but it has been suggested that this impression probably relates to patterns of patient referral to specialist centers [3].

In Japan, AIH predominantly affects women, occurring 6 times more frequently than in men, although the prevalence in men has been gradually increasing in recent years. AIH presentation is characterized by a monomodal peak after the age of 60 years, and AIH is frequently accompanied by other autoimmune diseases, including chronic thyroiditis, Sjögren's syndrome and rheumatoid arthritis. In Japan, the most frequently observed initial symptom is general malaise $(60 \%)$, followed by jaundice
(35\%) and appetite loss (27\%). Other primary symptoms include joint pain and fever (15\% each), both of which are rare in chronic viral hepatitis.

\section{Diagnostic Criteria}

Serological findings are the predominant elevation of liver enzymes over biliary enzymes, elevated serum IgG levels, and the presence of antibodies such as ANA, SMA and anti-LKM-1. Characteristic findings in the liver include interface hepatitis, infiltration of lymphocytes and plasma cells, and rosette formation. AIH is treated with steroids and immunosuppressive agents.

In Japan, AIH diagnostic criteria were established in 1996 by the Intractable Liver Disease Research Project Team of the Ministry of Health, Labor and Welfare (table 1) [4], which recommended the use of the IAIHG diagnostic criteria. The IAIHG diagnostic criteria were first established in 1993, revised in 1999, and are currently used as the international criteria (table 2) [3]. Characteristic AIH findings, including sex, clinical and serological features, liver histology and treatment response, are as- 
Table 2. IAHG scoring system for the diagnosis of AIH

\begin{tabular}{|c|c|}
\hline Parameters/features & Score \\
\hline Female & 2 \\
\hline \multicolumn{2}{|l|}{ ALP:AST (or ALT) ratio } \\
\hline$<1.5$ & 2 \\
\hline $1.5-3.0$ & 0 \\
\hline$>3.0$ & -2 \\
\hline \multicolumn{2}{|l|}{ Serum globulins or IgG above normal } \\
\hline$>2.0$ & 3 \\
\hline $1.5-2.0$ & 2 \\
\hline $1.0-1.5$ & 1 \\
\hline$<1.0$ & 0 \\
\hline \multicolumn{2}{|l|}{ ANA, SMA or anti-LKM-1 } \\
\hline$>1: 80$ & 3 \\
\hline $1: 80$ & 2 \\
\hline $1: 40$ & 1 \\
\hline$<1: 40$ & 0 \\
\hline AMA positive & -4 \\
\hline \multicolumn{2}{|l|}{ Hepatitis viral markers } \\
\hline Positive & -3 \\
\hline Negative & 3 \\
\hline \multicolumn{2}{|l|}{ Drug history } \\
\hline Positive & -4 \\
\hline Negative & 1 \\
\hline \multicolumn{2}{|l|}{ Average alcohol intake } \\
\hline$<25 \mathrm{~g} /$ day & 2 \\
\hline$>60$ g/day & -2 \\
\hline \multicolumn{2}{|l|}{ Liver histology } \\
\hline Interface hepatitis & 3 \\
\hline Predominantly lymphoplasmacytic infiltrate & 1 \\
\hline Rosetting of liver cells & 1 \\
\hline None of the above & -5 \\
\hline Biliary changes & -3 \\
\hline Other changes & -3 \\
\hline Other autoimmune disease(s) & 2 \\
\hline \multicolumn{2}{|l|}{ Optimal additional parameters } \\
\hline Seropositivity for other defined autoantibodies & 2 \\
\hline HLA-DR3 or DR4 & 1 \\
\hline \multicolumn{2}{|l|}{ Response to therapy } \\
\hline Complete & 2 \\
\hline Relapse & 3 \\
\hline \multicolumn{2}{|l|}{ Interpretation of aggregate scores } \\
\hline \multicolumn{2}{|l|}{ Pretreatment } \\
\hline Definite AIH & $>15$ \\
\hline Probable AIH & $10-15$ \\
\hline \multicolumn{2}{|l|}{ Post-treatment } \\
\hline Definite AIH & $>17$ \\
\hline Probable AIH & $12-17$ \\
\hline
\end{tabular}

Modified from Alvarez et al. [3]. AMA = Antimitochondrial antibody; ALP = alkaline phosphatase; AST = aspartate aminotransferase; HLA = human leukocyte antigen.
Table 3. Simplified diagnostic criteria for AIH

\begin{tabular}{lll}
\hline Variable & Cutoff & Points \\
\hline ANA or SMA & $\geq 1: 40$ & 1 \\
ANA or SMA & $\geq 1: 80$ & $2^{\mathrm{a}}$ \\
Or anti-LKM-1 & $\geq 1: 40$ & $2^{\mathrm{a}}$ \\
Or SLA & Positive & $2^{\mathrm{a}}$ \\
IgG & Above normal & 1 \\
& $\geq 1.1$ times upper & 2 \\
Liver histology (evidence or & Cormal limit & \\
hepatitis is a necessary condition) & Compatible with & 1 \\
Absence of viral hepatitis & Typical AIH & 2 \\
& Yes & 2 \\
\hline Total & Probable AIH & $\geq 6$ \\
& Definite AIH & $\geq 7$ \\
\hline
\end{tabular}

Modified from Hennes et al. [5]. SLA = Anti-soluble liver antigen. ${ }^{a}$ Addition of points achieved for all autoantibodies (maximum 2 points).

sessed using the scoring system, and definitive and probable diagnoses are compared before and after treatment. The criteria are complex and purely meant for scientific purposes. In 2008, the IAIHG introduced the simplified version of the AIH diagnostic criteria (table 3) [5] for routine clinical practice. This has made diagnosis easier and early therapeutic intervention possible. However, the diagnosis of AIH can be difficult because of its various clinical presentations, the presence of atypical and acute cases, and the involvement of other autoimmune diseases.

\section{Treatment}

Steroids and immunosuppressive drugs such as azathioprine are effective treatments for $\mathrm{AIH}$, and ursodeoxycholic acid has shown good efficacy in many studies.

\section{IgG4-Related Diseases}

AIP is known to be accompanied by multiple organ failure, including sclerosing cholangitis, liver failure, inflammation of the lacrimal and salivary glands, thyroiditis, interstitial pneumonia and interstitial nephritis. Systemic inflammatory diseases characterized by the infiltration of IgG4-positive plasma cells and elevated serum IgG4 levels were recently classified as IgG4-related disease. In 2011, the Ministry of Health, Labor and Welfare established the 2011 comprehensive diagnostic criteria for IgG4-related disease (table 4) [6]. 
Table 4. Comprehensive diagnostic criteria for IgG4-related disease, 2011

Concept IgG-related disease is a disease with an unknown cause and is characterized by synchronous or asynchronous swelling,
nodule formation and hypertrophic lesions in multiple organs due to tissue fibrosis and the marked infiltration of
lymphocytes and IgG4-positive plasma cells. The pancreas, biliary duct, lacrimal and salivary glands, central nervous
system, thyroid gland, lungs, liver, gastrointestinal tract, kidneys, prostate gland, retroperitoneum, arteries, lymph nodes,
skin and mammary gland are known to be affected. In general, the disease produces systemic manifestations, with some
cases of single organ involvement. Clinically, individual organs present different symptoms, with occasional severe
complications such as enlarged organs, obstruction caused by hypertrophic lesions, compression, infiltration and organ
dysfunction due to fibrosis. Corticosteroids are generally effective.

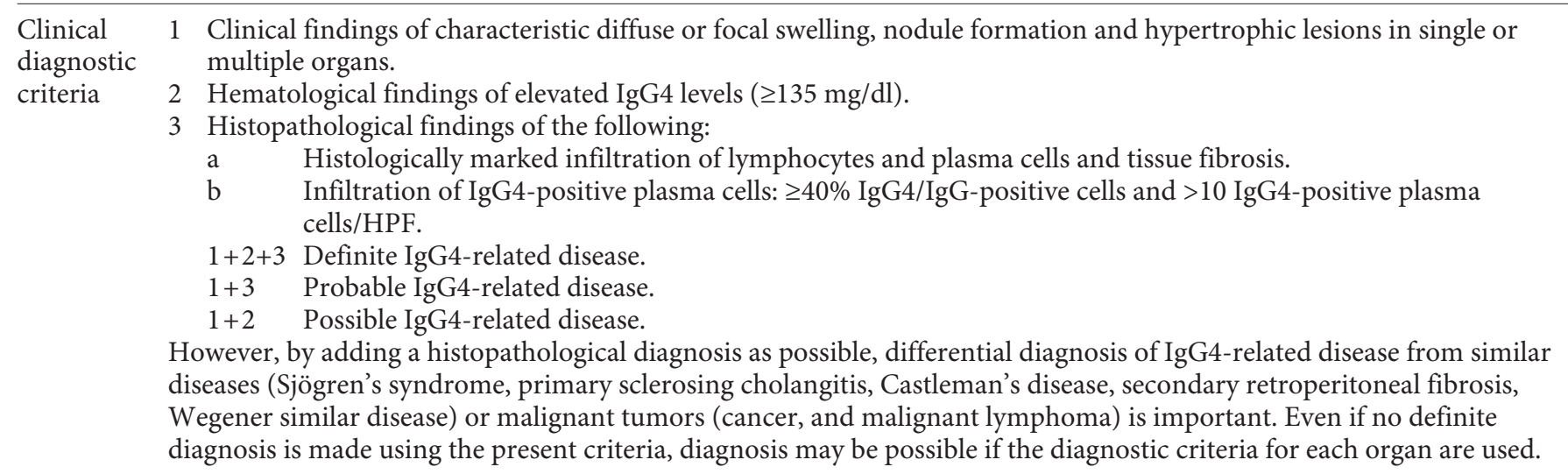

Modified from [6].

Liver injury is observed in 60-70\% of AIP cases; however, with the exception of obstructive jaundice, the cause of liver injury in AIH is not always clear. Umemura et al. [7] showed that AIP is accompanied by hepatocellular damage, such as the infiltration of IgG4-positive plasma cells near the portal vein, patterns of portal inflammation, large biliary duct damage, portal sclerosis, lobular hepatitis and cholestasis, and improvement in histological findings after steroid therapy. They called the disease IgG4 hepatopathy.

\section{AlH and IgG4-Associated AlH}

Recently, some AIH cases fulfilling the criteria of IgG4-related disease have been designated as IgG4-associated AIH $[8,9]$. Chung et al. [9] reported that a group of AIH patients with infiltration of IgG4-positive plasma cells were successfully treated with prednisolone (PSL) therapy. Based on the IgG4 immunoreactivity of liver biopsy samples ( $\geq 5$ IgG4-positive plasma cells/high-power field, HPF), they divided 26 patients with a definitive diagnosis of AIH into IgG4-positive (9 patients, 35\%) and IgG4-negative (17 patients, 65\%) groups (table 1). No pancreaticobiliary lesions were observed in the AIH patients. No IgG4-positive plasma cells were observed in 10 cases of primary biliary cirrhosis or 20 cases of chronic hepatitis C. The IgG4-positive group had a significantly higher level of serum IgG than the IgG4-negative group $(\mathrm{p}<0.01)$, but no significant differences in IgG4 levels were observed between these two groups. In addition, there were no significant differences in alanine aminotransferase (ALT), alkaline phosphatase, $\gamma$-glutamyl transpeptidase or ANA. On the other hand, the severity of plasma cell infiltration and lobular hepatitis were significantly high in the IgG4-positive group. Although portal inflammation and interface hepatitis were similar in both groups, the severity of portal inflammation was significantly higher in the IgG4-positive group. The infiltration of B cells, T cells and plasma cells was also significantly higher in the IgG4-positive group than in the IgG4negative group $(\mathrm{p}<0.05)$. Furthermore, ALT levels at 4 , 48, 72 and 96 weeks after the initiation of PSL therapy were significantly lower in the IgG4-positive group than in the IgG4-negative group (table 2). During the administration of PSL, hepatitis relapse was observed in 6 IgG4negative patients (35\%) but not in any IgG4-positive patients. Even with the definition of IgG4 positive as the 
infiltration of $\geq 10$ IgG4-positive plasma cells/HPF, the response to PSL therapy and the serum levels of IgG were significantly different between the groups.

Umemura et al. [8] also reported the pathology of IgG4-related AIH. In a study of $60 \mathrm{AIH}$ patients, they defined IgG4-related AIH as the infiltration of IgG4-positive plasma cells ( $\geq 10$ cells/HPF), IgG4-positive serum $(\geq 135 \mathrm{mg} / \mathrm{dl})$ and the ratio of IgG4 to IgG as 0.073 . However, because only 2 cases fulfilled this definition, they concluded that the prevalence of IgG4-positive AIH is extremely low (2/60 cases, $3.3 \%)$.

The main difference between the studies by Chung et al. [9] and Umemura et al. [8] is that, in the latter study, the definition included the ratio of IgG4 to IgG in addition to high serum IgG4 concentration $(\geq 135 \mathrm{mg} / \mathrm{dl})$ and the infiltration of IgG4-positive plasma cells in liver tissue. Furthermore, while Chung et al. [9] examined cases that matched the definite diagnosis of AIH, the $60 \mathrm{AIH}$ cases investigated by Umemura et al. [8] included 12 which were probable AIH. Moreover, the infiltration of IgG4-positive cells in the gallbladder and common bile duct was observed in 1 of the IgG4-associated AIH cases, indicating a case of IgG4-related sclerosing cholangitis rather than IgG4-associated AIH. Regardless of the differences, the two studies revealed that IgG4-related pathologies are associated with at least some AIH cases and that steroid treatment is effective in such IgG4-associated $\mathrm{AIH}$, as in other IgG4-related diseases.

\section{Conclusion}

Some patients with AIH present symptoms of IgG4related disease and respond effectively to steroid treatment. Although no unified diagnostic criteria are currently available, it is important to keep this disease in mind for definitive diagnosis and appropriate treatment. Further studies are needed to define the epidemiological features or diagnostic criteria.

\section{Disclosure Statement}

The authors have no conflicts of interest to declare.

\section{References}

$>1$ Mackay IR, Weiden S, Hasker J: Autoimmune hepatitis. Ann NY Acad Sci 1965;124:767780.

2 Johnson PJ, McFarlane IG: Meeting report: International Autoimmune Hepatitis Group. Hepatology 1993;18:998-1005.

-3 Alvarez F, Berg PA, Bianchi FB, et al: International Autoimmune Hepatitis Group Report: review of criteria for diagnosis of autoimmune hepatitis. J Hepatol 1999;31:929-938.

4 Research Program of Intractable Disease provided by the Ministry of Health Labor, and
Welfare of Japan: Guidelines for the diagnosis of autoimmune hepatitis, 1996 (in Japanese). Kanzo 1996;37:298-300.

$\checkmark 5$ Hennes EM, Zeniya M, Czaja AJ, et al: Simplified criteria for the diagnosis of autoimmune hepatitis. Hepatology 2008;48:169-176.

6 Research Program of Intractable Disease provided by the Ministry of Health Labor, and Welfare of Japan: Comprehensive diagnostic criteria for IgG4-related disease (IgG4-RD), 2011 (in Japanese). Nihon Naika Gakkai Zasshi 2012;101:795-804.
7 Umemura T, Zen Y, Hamano H, et al: Immunoglobin G4-hepatopathy: association of immunoglobin G4-bearing plasma cells in liver with autoimmune pancreatitis. Hepatology 2007;46:463-471.

8 Umemura T, Zen Y, Hamano H, et al: Clinical significance of immunoglobulin G4-associated autoimmune hepatitis. J Gastroenterol 2011;46(suppl 1):48-55.

>9 Chung $\mathrm{H}$, Watanabe T, Kudo M, et al: Identification and characterization of IgG4-associated autoimmune hepatitis. Liver Int 2010;30:222-231. 